\title{
Supplementary Data for: Discovery of mafic impact melt in the center of the Vredefort Dome; archetype for continental residua of Early Earth cratering?
}

\section{METHODS}

Optical and electron microscopy (secondary and backscatter electron imaging (SE and BSE), energy dispersive spectroscopy (EDS), cathodoluminescence (CL) and electron backscatter diffraction (EBSD)) of petrographic thin sections was carried out, using a Hitachi SU6600 Field Emission Gun-Scanning Electron Microscope (FEG-SEM) at the Western University Zircon and Accessory Phase Laboratory (ZAPLab). Zircon separation for geochronology was conducted at the Jack Satterly Geochronology lab at the University of Toronto using standard procedures. Secondary Ion Mass Spectrometer (SIMS) U-Pb isotopic analysis and Ti-thermometry was conducted at the Stanford/ U.S.G.S. SHRIMP-RG facility according to previously published procedures (Mazdab and Wooden, 2006), and referenced to internal zircon geochronology standard VP-10 (see Bowman et al., 2011). Lu-Hf isotope measurements of zircon were made by LA-MC-ICP-MS at the University of Bristol according to previously published procedures (Hawkesworth and Kemp, 2006; Fisher et al., 2011). The standards used were Plešovice (Sláma et al., 2008) which had an average ${ }^{176} \mathrm{Hf} /{ }^{177} \mathrm{Hf}$ of 0.282487 $\pm 0.000023(\mathrm{n}=20)$, Mud Tank (Woodhead and Hergt, 2005) with an average of $0.282523 \pm$ $0.000021(\mathrm{n}=19)$ and Temora-2 (Woodhead and Hergt, 2005) which had an average of $0.282700 \pm 0.000044(\mathrm{n}=9)$. 
Table DR1: U-Pb Data for V250, V232 and V235

\begin{tabular}{|c|c|c|c|c|c|c|c|c|c|c|c|c|c|c|c|c|c|}
\hline Sample \# & $\begin{array}{c}207 / 206 \\
\text { age }\end{array}$ & $\begin{array}{l}\text { 2sd } \\
\text { error }\end{array}$ & $\begin{array}{c}\text { Conc } \\
(\%)\end{array}$ & $\begin{array}{c}204 \\
\text { cts/sec }\end{array}$ & $204 / 206$ & $\begin{array}{l}\mathbf{P b} / \mathbf{U}: \\
\mathbf{U O} / \mathbf{U}^{2}\end{array}$ & $\begin{array}{c}\% \\
\text { err }\end{array}$ & $\begin{array}{c}\mathrm{Pb}^{204} \\
\text { Corr } \\
207 \mathbf{r} / 206 r\end{array}$ & $\begin{array}{c}\% \\
\text { err }\end{array}$ & $\begin{array}{c}\mathrm{Pb}^{204} \\
\mathrm{Corr} \\
207 \mathrm{r} / 23 \\
5 \mathrm{r}\end{array}$ & $\begin{array}{c}\% \\
\text { err }\end{array}$ & $\begin{array}{c}\mathrm{Pb}^{204} \\
\text { Corr } \\
207 \mathbf{r} / 238\end{array}$ & $\begin{array}{c}\% \\
\text { err }\end{array}$ & Err corr & $\begin{array}{c}\mathbf{U} \\
(\mathbf{p p m})\end{array}$ & $\begin{array}{c}\text { Th } \\
\text { (ppm) }\end{array}$ & $\mathbf{T h} / \mathbf{U}$ \\
\hline \multicolumn{18}{|l|}{ V09_232 } \\
\hline V09_232_1.1 & 1993 & 46 & 2 & 0.06 & $8.5 \mathrm{E}-5$ & .02779 & 1.1 & .1225 & 1.3 & 5.99 & 1.7 & .3547 & 1.1 & .653 & 37 & 17 & 0.49 \\
\hline V09_232_2.1 & 1984 & 56 & 0 & 0.07 & $1.3 \mathrm{E}-4$ & .02828 & 1.3 & .1219 & 1.6 & 6.06 & 2.1 & .3606 & 1.3 & .637 & 27 & 10 & 0.38 \\
\hline V09_232_3.1 & 2000 & 40 & 0 & 0.05 & $5.0 \mathrm{E}-5$ & .02845 & 1.0 & .1230 & 1.1 & 6.16 & 1.5 & .3632 & 1.0 & .670 & 53 & 27 & 0.53 \\
\hline V09_232_4.1 & 2013 & 40 & 0 & -0.07 & $-7.1 \mathrm{E}-5$ & .02874 & 1.0 & .1239 & 1.1 & 6.28 & 1.5 & .3676 & 1.0 & .649 & 51 & 26 & 0.52 \\
\hline V09_232_5.1 & 1995 & 60 & 1 & 0.05 & $1.0 \mathrm{E}-4$ & .02822 & 1.3 & .1227 & 1.7 & 6.09 & 2.1 & .3600 & 1.3 & .621 & 33 & 11 & 0.35 \\
\hline $\begin{array}{c}\text { V09_232_ } \\
7.1\end{array}$ & 2035 & 44 & 2 & 0.06 & $8.1 \mathrm{E}-5$ & .02830 & 1.1 & .1255 & 1.3 & 6.25 & 1.7 & .3611 & 1.1 & .661 & 48 & 38 & 0.82 \\
\hline V09_232_8.1 & 2003 & 36 & 1 & -0.13 & $-9.3 \mathrm{E}-5$ & .02807 & 0.8 & .1232 & 1.0 & 6.10 & 1.3 & .3591 & 0.8 & .633 & 81 & 41 & 0.51 \\
\hline \multicolumn{18}{|l|}{ V09_235 } \\
\hline V09_235_1.1 & 1994 & 30 & -1 & 0.10 & $5.5 \mathrm{E}-5$ & .02888 & 0.7 & .1225 & 0.9 & 6.23 & 1.1 & .3687 & 0.7 & .630 & 111 & 49 & 0.46 \\
\hline V09_235_2.1 & 2015 & 28 & 1 & 0.07 & $3.8 \mathrm{E}-5$ & .02839 & 0.7 & .1240 & 0.8 & 6.20 & 1.1 & .3625 & 0.7 & .666 & 106 & 48 & 0.46 \\
\hline V09_235_3.1 & 2089 & 74 & 3 & -0.21 & $-3.6 \mathrm{E}-4$ & .02885 & 1.4 & .1293 & 2.1 & 6.61 & 2.6 & .3706 & 1.5 & .570 & 34 & 14 & 0.42 \\
\hline V09_235_4.1 & 2025 & 34 & 4 & 0.03 & $2.5 \mathrm{E}-5$ & .02747 & 0.9 & .1248 & 1.0 & 6.04 & 1.3 & .3509 & 0.9 & .655 & 90 & 54 & 0.62 \\
\hline V09_235_5.1 & 2018 & 52 & 8 & 0.04 & $7.2 \mathrm{E}-5$ & .02628 & 1.2 & .1243 & 1.4 & 5.75 & 1.9 & .3355 & 1.2 & .643 & 48 & 21 & 0.45 \\
\hline V09_235_6.1 & 2003 & 32 & 2 & 0.06 & $4.1 \mathrm{E}-5$ & .02787 & 0.8 & .1232 & 0.9 & 6.04 & 1.2 & .3558 & 0.8 & .655 & 106 & 62 & 0.61 \\
\hline V09_235_7.1 & 2015 & 26 & 3 & 0.00 & --- & .02783 & 0.7 & .1240 & 0.7 & 6.08 & 1.0 & .3556 & 0.7 & .681 & 140 & 81 & 0.60 \\
\hline V09_235_8.1 & 2015 & 30 & 3 & 0.09 & $5.6 \mathrm{E}-5$ & .02773 & 0.7 & .1240 & 0.8 & 6.05 & 1.1 & .3541 & 0.7 & .653 & 117 & 46 & 0.40 \\
\hline V09_235_9.1 & 2005 & 40 & 8 & 0.08 & $8.3 \mathrm{E}-5$ & .02628 & 0.9 & .1234 & 1.1 & 5.70 & 1.5 & .3353 & 0.9 & .636 & 93 & 54 & 0.60 \\
\hline & & & & & & & & & & & & & & & & & \\
\hline \multicolumn{18}{|l|}{ V09_250 } \\
\hline V09_250_1.1 & 2009 & 48 & 2 & 0.06 & 7.1E-5 & .02802 & 1.0 & .1236 & 1.4 & 6.10 & 1.7 & .3576 & 1.0 & .613 & 50 & 17 & 0.36 \\
\hline V09_250_4.1 & 2030 & 50 & 1 & 0.00 & --- & .02864 & 1.3 & .1251 & 1.4 & 6.31 & 1.9 & 3659 & 1.3 & .691 & 29 & 7 & 0.24 \\
\hline V09_250_5.1 & 2016 & 38 & 1 & 0.00 & --- & 0.2843 & 1.0 & .1241 & 1.1 & 6.22 & 1.5 & .3633 & 1.0 & .685 & 59 & 17 & 0.30 \\
\hline
\end{tabular}


Table DR2: Ti-in-zircon data

\begin{tabular}{|c|c|c|c|}
\hline Sample & Ti 48(ppm) & Ti 49(ppm) & $\mathbf{T}\left({ }^{\circ} \mathrm{C}\right)$ \\
\hline V09-232-1.3TE & 19.1 & 19.2 & 833 \\
\hline V09-232-2.2TE & 30.1 & 30.3 & 883 \\
\hline V09-232-2.3TE & 22.7 & 22.9 & 852 \\
\hline V09-232-3.2TE & 27.1 & 27.8 & 873 \\
\hline V09-232-3.3TE & 17.8 & 19.0 & 832 \\
\hline V09-232-4.2TE & 32.5 & 32.2 & 890 \\
\hline V09-232-5.2TE & 24.0 & 23.9 & 857 \\
\hline V09-232-5.3TE & 13.2 & 13.2 & 795 \\
\hline V09-232-6.2TE & 38.4 & 38.2 & 910 \\
\hline V09-232-6.3TE & 30.8 & 31.9 & 889 \\
\hline V09-232-7.2TE & 29.4 & 27.8 & 874 \\
\hline V09-232-7.3TE & 18.2 & 17.5 & 824 \\
\hline V09-232-8.2TE & 15.2 & 14.4 & 804 \\
\hline V09-232-9.4TE & 31.5 & 31.2 & 887 \\
\hline V09-232-9.5TE & 30.5 & 30.8 & 885 \\
\hline V09-232-9.6TE & 32.7 & 34.0 & 897 \\
\hline V09-235-1.2TE & 18.6 & 18.9 & 832 \\
\hline V09-235-2.2TE & 15.8 & 15.6 & 812 \\
\hline V09-235-3.2TE & 44.9 & 44.1 & 928 \\
\hline V09-235-4.2TE & 18.7 & 18.3 & 828 \\
\hline V09-235-5.2TE & 26.8 & 27.2 & 871 \\
\hline V09-235-6.2TE & 22.7 & 21.9 & 847 \\
\hline V09-235-7.2TE & 19.0 & 19.0 & 832 \\
\hline V09-235-8.2TE & 18.0 & 17.5 & 824 \\
\hline V09-235-9.2TE & 20.8 & 20.9 & 842 \\
\hline V09-235-9.3TE & 29.4 & 29.6 & 880 \\
\hline V09-235-10.2TE & 18.0 & 17.5 & 824 \\
\hline V09-250-1.2TE & 22.7 & 22.3 & 849 \\
\hline V09-250-2.2TE & 19.1 & 19.9 & 837 \\
\hline V09-250-3.2TE & 45.0 & 42.9 & 924 \\
\hline V09-250-4.2TE & 18.3 & 17.9 & 826 \\
\hline V09-250-5.2TE & 23.4 & 22.5 & 850 \\
\hline V09-250-6.2TE & 27.1 & 26.3 & 867 \\
\hline V09-250-6.3TE & 24.0 & 24.2 & 858 \\
\hline
\end{tabular}


Table DR3: Lu-Hf data

\begin{tabular}{|c|c|c|c|c|c|c|c|}
\hline Name & $\begin{array}{c}\text { Age Ma } \\
\pm 1 \sigma\end{array}$ & ${ }^{176} \mathrm{Lu} /{ }^{177} \mathrm{Hf}$ & ${ }^{176} \mathrm{Yb} /{ }^{177} \mathbf{H f}$ & ${ }^{176} \mathrm{Hf} /{ }^{177} \mathrm{Hf}$ & $\pm 1 \sigma$ & $\varepsilon_{\mathrm{HfT}} \pm 2 \sigma$ & $\begin{array}{c}\text { T(DM) } \\
\text { Ma }^{\mathbf{c}}\end{array}$ \\
\hline V250- Z1 & 2020 & 0.000595 & 0.025532 & 0.281345 & 0.000017 & -5.31 .2 & 2993 \\
\hline V250- Z2 & 2020 & 0.000858 & 0.032652 & 0.281435 & 0.000019 & -2.11 .3 & 2791 \\
\hline V250- Z6 & 2020 & 0.000549 & 0.023084 & 0.281407 & 0.000014 & -3.11 .0 & 2853 \\
\hline V250- Z9 & 2020 & 0.000439 & 0.017480 & 0.281426 & 0.000011 & -2.40 .8 & 2811 \\
\hline V250- Z10 & 2020 & 0.000556 & 0.023501 & 0.281454 & 0.000016 & -1.41 .1 & 2748 \\
\hline V250-Z11 & 2020 & 0.000389 & 0.015887 & 0.281443 & 0.000012 & -1.80 .9 & 2772 \\
\hline V250-Z12 & 2700 & 0.001548 & 0.064351 & 0.281060 & 0.000015 & 0.41 .1 & 3166 \\
\hline V250-Z13 & 3200 & 0.001188 & 0.048438 & 0.281036 & 0.000013 & 11.41 .0 & 2855 \\
\hline V235-Z15 & 2020 & 0.000689 & 0.027850 & 0.281296 & 0.000011 & -7.00 .8 & 3104 \\
\hline V235-Z16 & 2020 & 0.000560 & 0.022015 & 0.281290 & 0.000015 & $\begin{array}{ll}-7.2 & 1.0\end{array}$ & 3116 \\
\hline V235- Z20 & 2020 & 0.000398 & 0.015387 & 0.281342 & 0.000020 & -5.41 .4 & 3000 \\
\hline V235- Z21 & 2020 & 0.000445 & 0.016924 & 0.281317 & 0.000016 & -6.31 .2 & 3057 \\
\hline V235- Z25 & 2020 & 0.000281 & 0.010548 & 0.281292 & 0.000015 & -7.21 .1 & 3112 \\
\hline V235- Z26 & 2020 & 0.000652 & 0.024328 & 0.281329 & 0.000013 & -5.80 .9 & 3028 \\
\hline V235- Z27 & 2020 & 0.000529 & 0.020668 & 0.281286 & 0.000015 & -7.41 .0 & 3126 \\
\hline V235- Z30 & 2020 & 0.000418 & 0.016673 & 0.281272 & 0.000020 & $\begin{array}{ll}-7.9 & 1.4\end{array}$ & 3158 \\
\hline
\end{tabular}

${ }^{\mathrm{c}}$ Stands for crustal source. 
Figure DR1: MicroGIS of thin section V235 with distribution of zircons and baddeleyites, grain size is indicated by spot size.

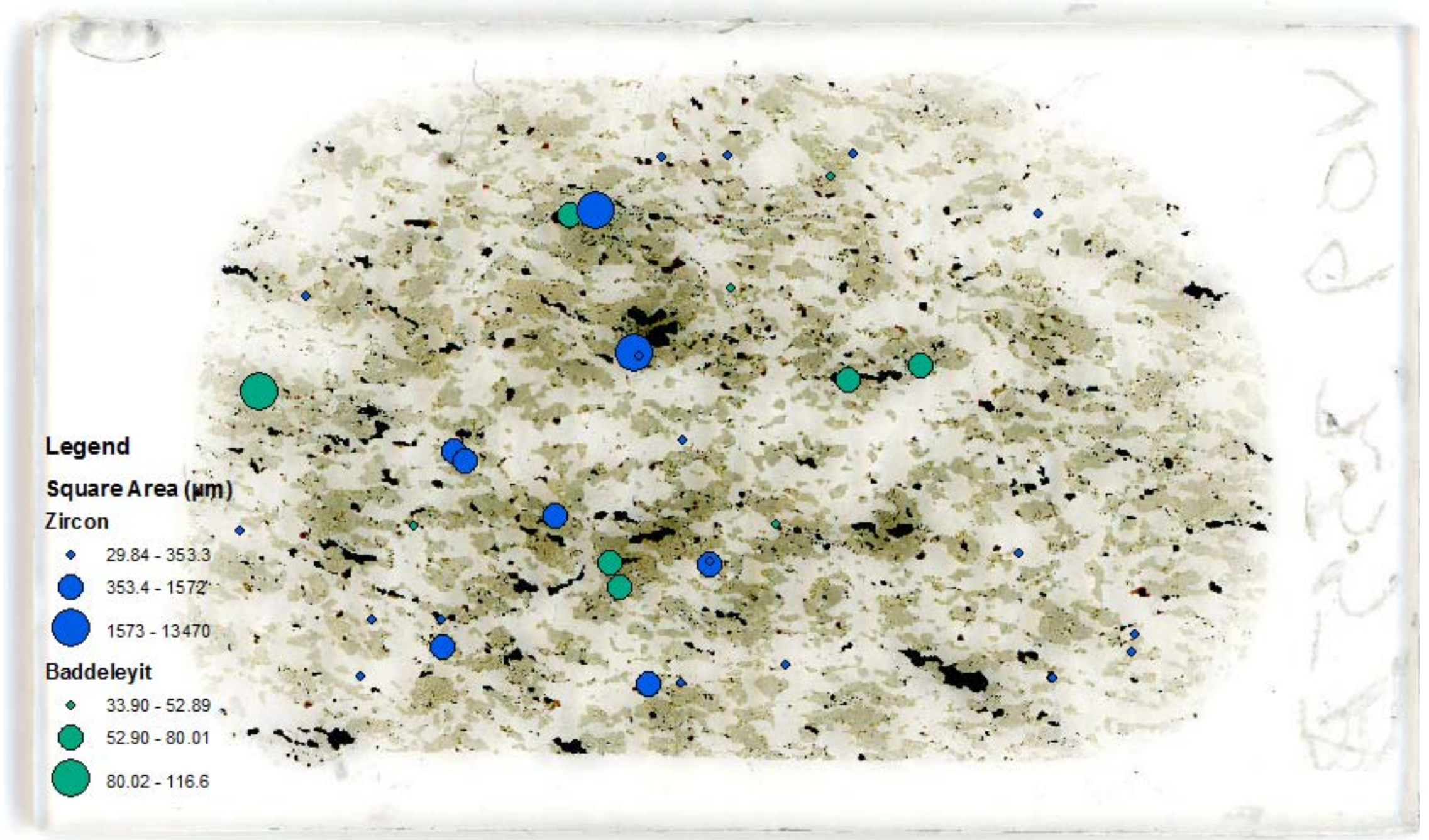




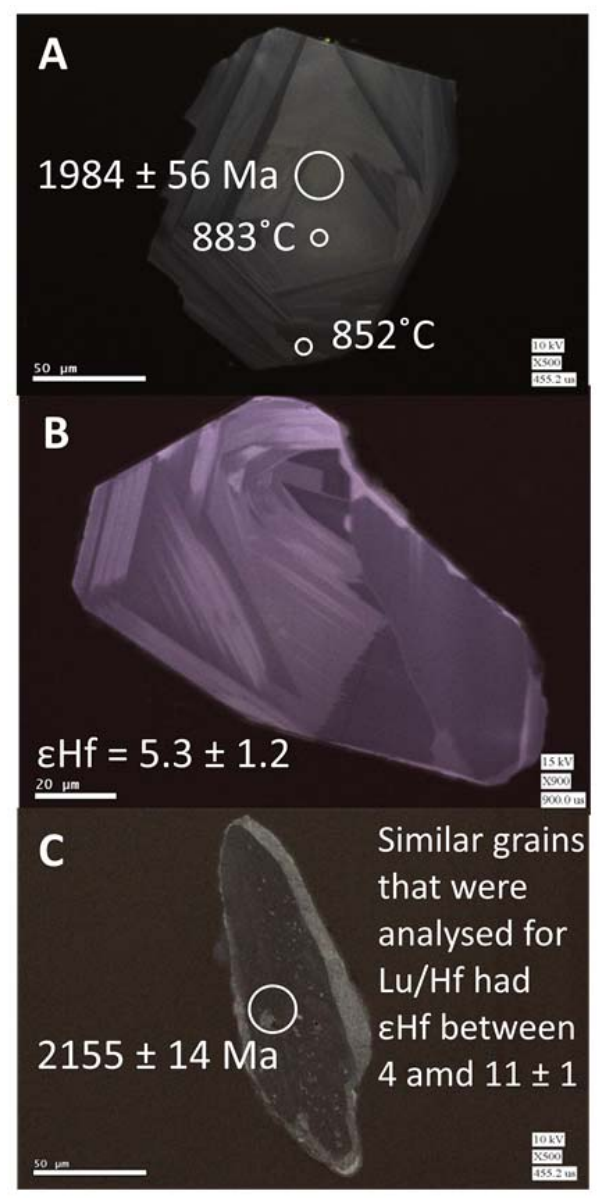

Fig. 2: $\mathrm{CL}$ images of zircons from the gabbronorite body, polished to mid-plane and imaged by FEG-SEM.: (A) CL image of unshocked, igneous grain with typical oscillatory planar growth banding and sector zoning are from sample V232 at Site 2. Note the clearly different $\mathrm{CL}$ zoning patterns in the shocked and unshocked grains. This grain has a $\mathrm{U}$-pb age of $1984 \pm 56 \mathrm{Ma}$ and has a core temperature of $883^{\circ} \mathrm{C}$ and a rim temperature of $852^{\circ} \mathrm{C}$. (B) $\mathrm{CL}$ image of an unshocked, igneous grain from $\mathrm{V} 250$ that was analysed for $\mathrm{Lu} / \mathrm{Hf}$ and has a $\varepsilon \mathrm{Hf}$ value of $-5.3 \pm 1.2$. (C) $\mathrm{CL}$ image illustrates a shocked and recrystallized xenocrystic grain from sample V250 from site 1 , this grain has a U-Pb age of $2155 \pm 14 \mathrm{Ma}$. Similar grains from this sample were analysed for $\mathrm{Lu} / \mathrm{Hf}$ ratios and have $\varepsilon \mathrm{Hf}$ values between 4 and $11 \pm 1$. 


\section{REFERENCES}

Bowman, J.R., Moser, D.E., Valley, J.W., Wooden, J.L., Kita, N.T., and Mazdab, F.K., 2011, Zircon U$\mathrm{Pb}$ isotopic, $\delta^{18} \mathrm{O}$ and trace element response to $80 \mathrm{~m}$.y. of high temperature metamorphism in the lower crust: sluggish diffusion and new records of Archean craton formation: American Journal of Science, v. 311, p. 719-772, DOI 10.2475/09.2011.01.

Fisher, C.M., Hanchar, J.M., Samson, S.D., Dhuime, B., Blichert-Toft, J., Vervoort, J.D., and Lam, R., 2011, Synthetic zircon doped with hafnium and rare earth elements: A reference material for in situ hafnium isotope analysis: Chemical Geology, v. 286, p. 32-47 doi:10.1016/j.chemgeo.2011.04.013.

Hawkesworth ,C.J., and Kemp, A.I.S., 2006, Using hafnium and oxygen isotopes in zircons to unravel the record of crustal evolution: Chemical Geology, v. 226, p. 144-162, doi:10.1016/j.chemgeo.2005.09.018 13.

Mazdab, F.M., and Wooden, J.L.,2006, Trace element analysis in zircon by ion microprobe (SHRIMP$\mathrm{RG}$ ); technique and applications:Geochimica et Cosmochimica Acta, v. 70, Supp.1, p. A405, doi:10.1016/j.gca.2006.06.817.

Sláma, J., Košler, J., Condon, D. J., Crowley, J. L., Gerdes, A., Hanchar, J. M., Horstwood, M. S. A., Morris, G. A., Nasdala, L., Norberg, N., Schaltegger, U., Schoene, B., Tubrett, M. N., and Whitehouse, M. J., 2008, Plešovice zircon - A new natural reference material for U-Pb and $\mathrm{Hf}$ isotopic microanalysis: Chemical Geology, v. 249, p. 1-35.

Woodhead, J. D., and Hergt, J. M., 2005, A Preliminary Appraisal of Seven Natural Zircon Reference Materials for In Situ Hf Isotope Determination: Geostandards and Geoanalytical Research, v. 29, p. 183-195. 